\title{
An Indolent Presentation of Gliomatosis Cerebri in an Elderly Patient: Posterior Fossa Decompression Prior to Treatment
}

Young Sill Kang ${ }^{1}$, Ami Raval ${ }^{2}$, Karen S. Black ${ }^{3}$, Jian Yi Li ${ }^{4}$, Richard H. Blanck ${ }^{5}$, Michael Schulder 6

1. Charité Medical School, Charité School of Medicine and University Hospital, Berlin, Germany 2. Department of Neurosurgery, Staten Island University Hospital 3. Division of Neuroradiology, Department of Radiology, Hofstra North Shore-LIJ School of Medicine, North Shore University Hospital 4. Department of Pathology and Lab Medicine, Hofstra North Shore-LIJ School of Medicine, North Shore University Hospital 5. Department of Neurology, Hofstra North Shore-LIJ School of Medicine, North Shore University Hospital 6. School of Medicine, Hofstra University

$\square$ Corresponding author: Michael Schulder, schulder@nshs.edu Disclosures can be found in Additional Information at the end of the article

\section{Abstract}

Gliomatosis cerebri (GC) is a rare astrocytic tumor that typically affects multiple lobes of the brain. Due to its insidious nature and atypical symptom presentation, diagnosis is often made late in the course of disease process. To date, no definitive treatment guidelines have been established, and due to its infiltrating pattern, neurosurgical resection of the lesion is generally not feasible. We report a case of a 69-year-old male who initially presented with symptoms similar to normal pressure hydrocephalus. The patient underwent CSF diversion, with an inconclusive tissue biopsy. His symptoms were relieved for more than two years thereafter until he demonstrated worsening of symptoms, with a MRI clearly demonstrating GC. Patients presenting with hydrocephalus and a "full posterior fossa" should be followed carefully for evidence of GC progression.

Review began 06/01/2014 Published 11/17/2014

C) Copyright 2014

Kang et al. This is an open access article distributed under the terms of the Creative Commons Attribution License CC-BY 3.0., which permits unrestricted use, distribution, and reproduction in any medium, provided the original author and source are credited.
Categories: Neurology, Neurosurgery, Oncology

Keywords: gliomatosis cerebri, biopsy, shunt, glioma, posterior fossa decompression, infiltrative glioma

\section{Introduction}

Gliomatosis cerebri (GC) is an uncommon tumor of the central nervous system, which histologically resembles a diffuse cerebral infiltration by glial neoplasm without formation of a solid tumor mass and preserves the architecture of the tissue surrounding the tumor [1]. The current World Health Organization (WHO) classification defines GC as a highly infiltrative glial lesion that affects at least three cerebral lobes [2], usually including bilateral hemispheres and/or deep gray matter [1-2]. GC can simultaneously infiltrate multiple sites in the central nervous system, including the cerebrum, cerebellum, brainstem, and spinal cord [3]. Because of its infiltrative character, it is known that surgical treatment is generally not applicable. Radiotherapy and adjuvant chemotherapy with various agents have been suggested for disease control, and recently, positive treatment responses were demonstrated in published literature [4]. However, additional retrospective and prospective clinical trials are needed to support the positive outcomes. Due to the limited number of patients that have been treated with radiotherapy alone, a statistically significant association between radiotherapy and survival has not been shown. Presenting common symptoms, such as headache, seizures, and muscle 
weakness, are unspecific and often delay diagnosis. All of these factors make the prognosis poor. Patient demographics show a wide range in age at presentation of GC (9 to 87 years) but the majority of patients are comparatively young (median age: 46 years) [5].

We discuss the presentation and diagnosis of GC in an elderly patient. Exhibited symptoms at the time of initial presentation were unspecific and were demonstrative more of normal pressure hydrocephalus, which delayed the final diagnosis. This case demonstrates that, in an elderly patient who presents with gait instability and evidence of a diffuse unspecific lesion within the posterior fossa, he/she must be followed carefully for evolution of symptomatology for evidence of occult development of pathology, such as gliomatosis cerebri.

\section{Case Presentation}

A 69-year-old man with a past medical history significant only for hypothyroidism presented with a one-year history of gait disturbance, resulting in multiple falls. The family noted mild cognitive deterioration as well, with increasing urinary frequency. The patient denied any headache or visual changes.

\section{Consent}

Written and verbal informed consent has been obtained for publication.

\section{Examination}

On examination, the patient was awake and talkative with recall of two out of three objects at five minutes. Cranial nerves were all intact. Strength, tone, and reflexes were symmetric and normal in all extremities. Gait examination revealed a steady but slightly halting walk with inability to tandem walk without Romberg's sign. No frontal release signs were present.

\section{Imaging}

MRI scan of the brain demonstrated hydrocephalus with subtle fullness in the posterior fossa, and T2/FLAIR hyperintensities surrounding both lateral ventricles, particularly within the parietal and temporal regions (Figure 1). MRI scan of the spine demonstrated only subtle enlargement in cord contour with a slight increase in the cord signal intensity on short TI inversion recovery (STIR) sequences in upper and mid-cervical spines. 


\section{Cureus}
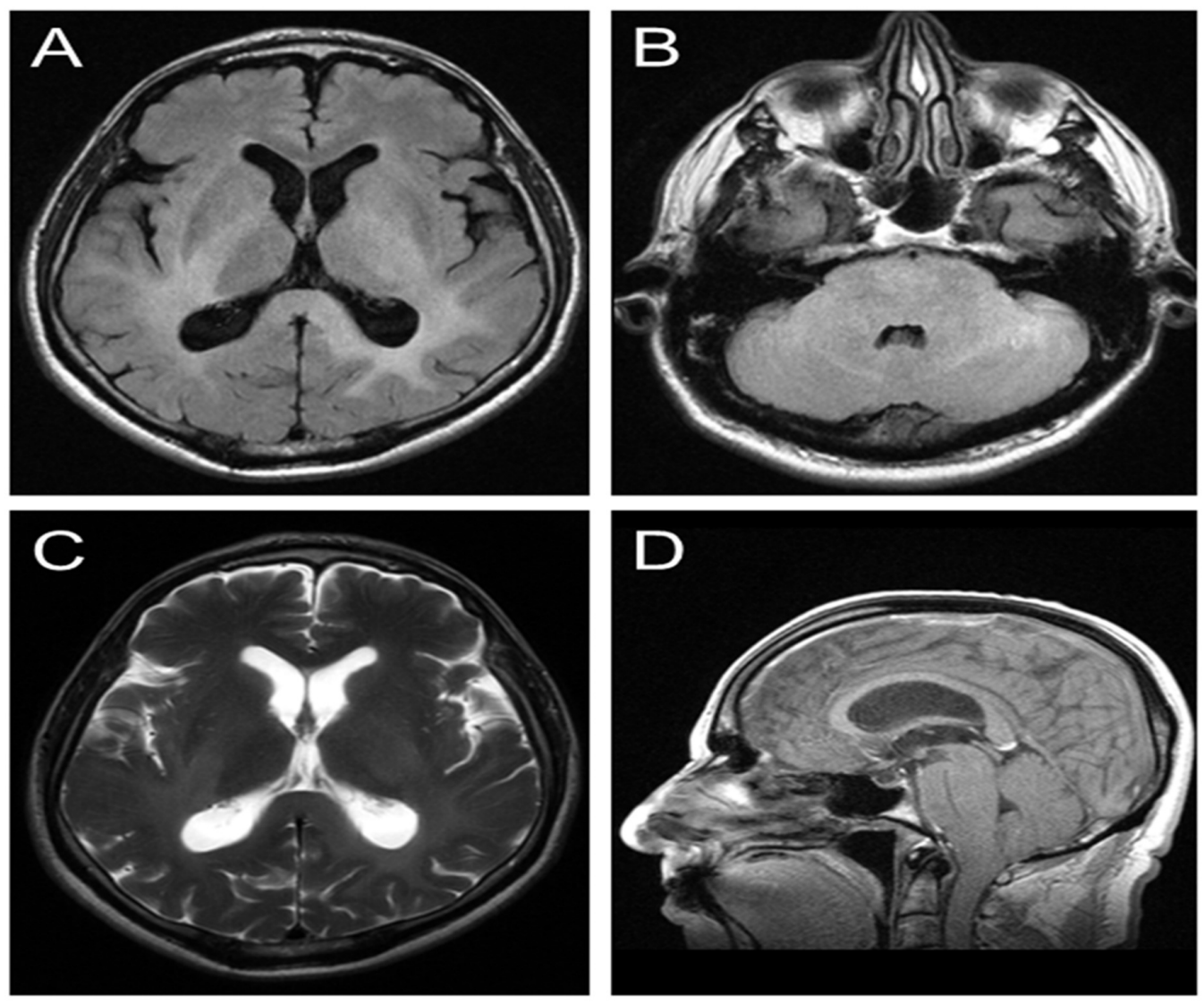

FIGURE 1:

Initial images upon presentation. (A) FLAIR MR axial images of supratentorium. (B) FLAIR MR axial images of cerebellum and brainstem. (C) T2 weighted MR images. (D) T1 weighted MR sagittal images demonstrated downward displacement of cerebellar tonsils.

The patient underwent a ventriculoperitoneal shunt. At the time of the shunt placement, CSF as well as intraparenchymal tissue along the path of the ventricular catheter was obtained for pathology in order to rule out an infectious, inflammatory, or demyelinating process.

Pathologic findings demonstrated minimal reactive astrogliosis. Immunostains were negative for p53, CD3, and CD 20, with a very low MIB-1 (Ki-67). CSF cytopathologic findings were negative for malignant cells.

\section{Postoperative course}

After shunt placement, the patient's symptoms significantly improved. Follow-up imaging studies performed 6, 12 (Figure 2), and 18 months from the initial ones demonstrated decrease in ventricular size with stable T2/FLAIR changes in the brainstem and cerebellum without any evidence of evolving pathology. 


\section{Cureus}
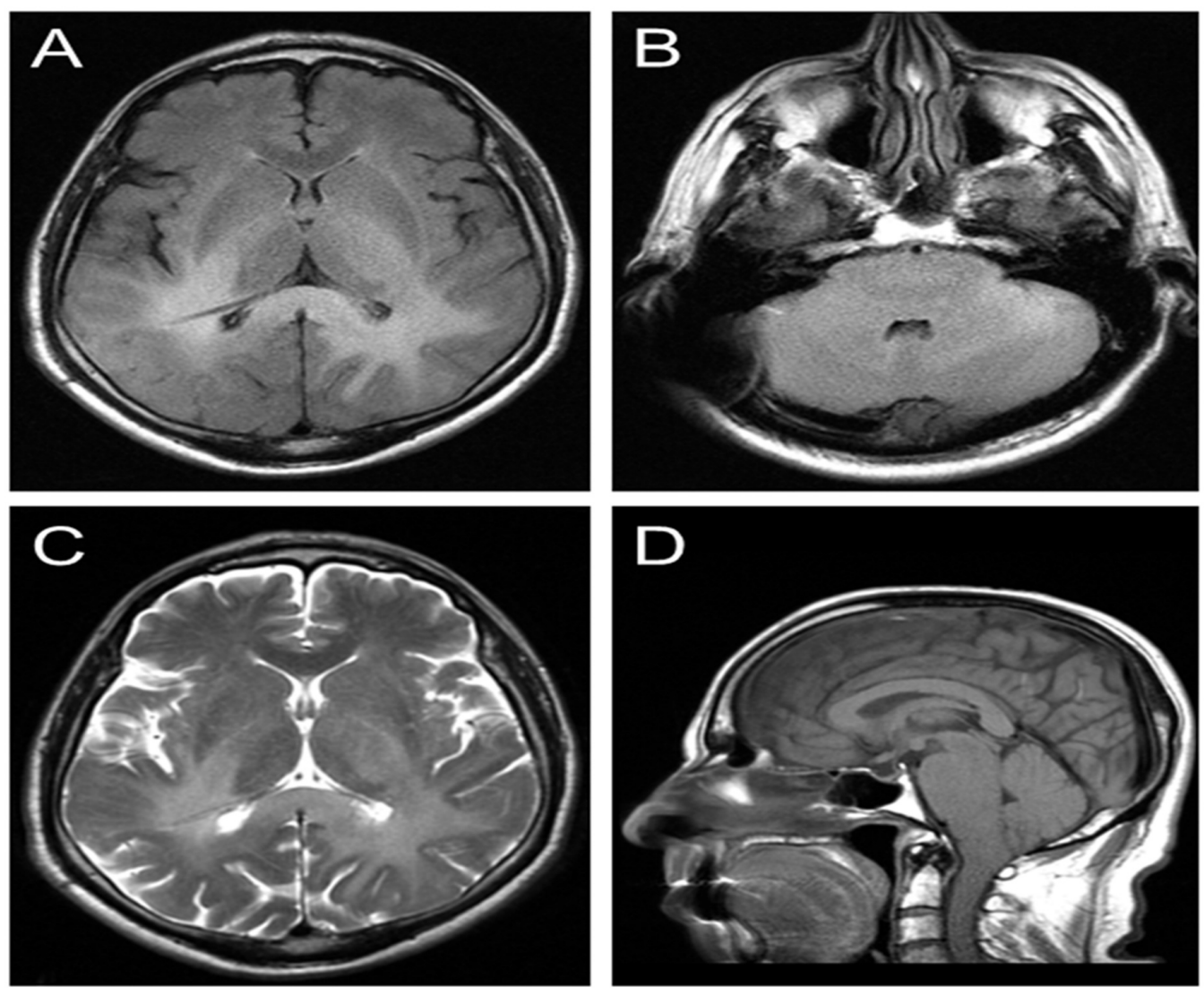

\section{FIGURE 2: Follow-up images}

Follow-up images performed 12 months after initial one performed on presentation and 10 months after right parietal ventriculoperitonal shunt placement. (A) FLAIR MR axial images of supratentorium. Ventricular size has decreased, with no significant change in FLAIR and T2 hyperintensities. (B) FLAIR MR axial images of cerebellum and brainstem. (C) T2 weighted MR images. (D) T1 weighted MR sagittal images demonstrating the stable downward displacement of cerebellar tonsils.

Approximately two years after initial presentation, the patient's gait difficulty started to slowly deteriorate, starting from increasing frequency of falls to where patient ultimately was unable to ambulate unassisted. Examination revealed a wide-based gait and dysarthria with moderate ataxia and postural instability. The patient was unable to tandem walk and demonstrated a Romberg's sign. Cranial nerves and motor examination were unrevealing. Shunt interrogation demonstrated the shunt to be working properly as well. Repeat MRI demonstrated stable findings compared with prior MRIs with abnormal T2 signal hyperintensity within the cerebral hemispheres, brainstem, and cerebellum without gadolinium enhancement. There was persistent, but unchanged, downward displacement of the cerebellar tonsils (Figure 3). 


\section{Cureus}
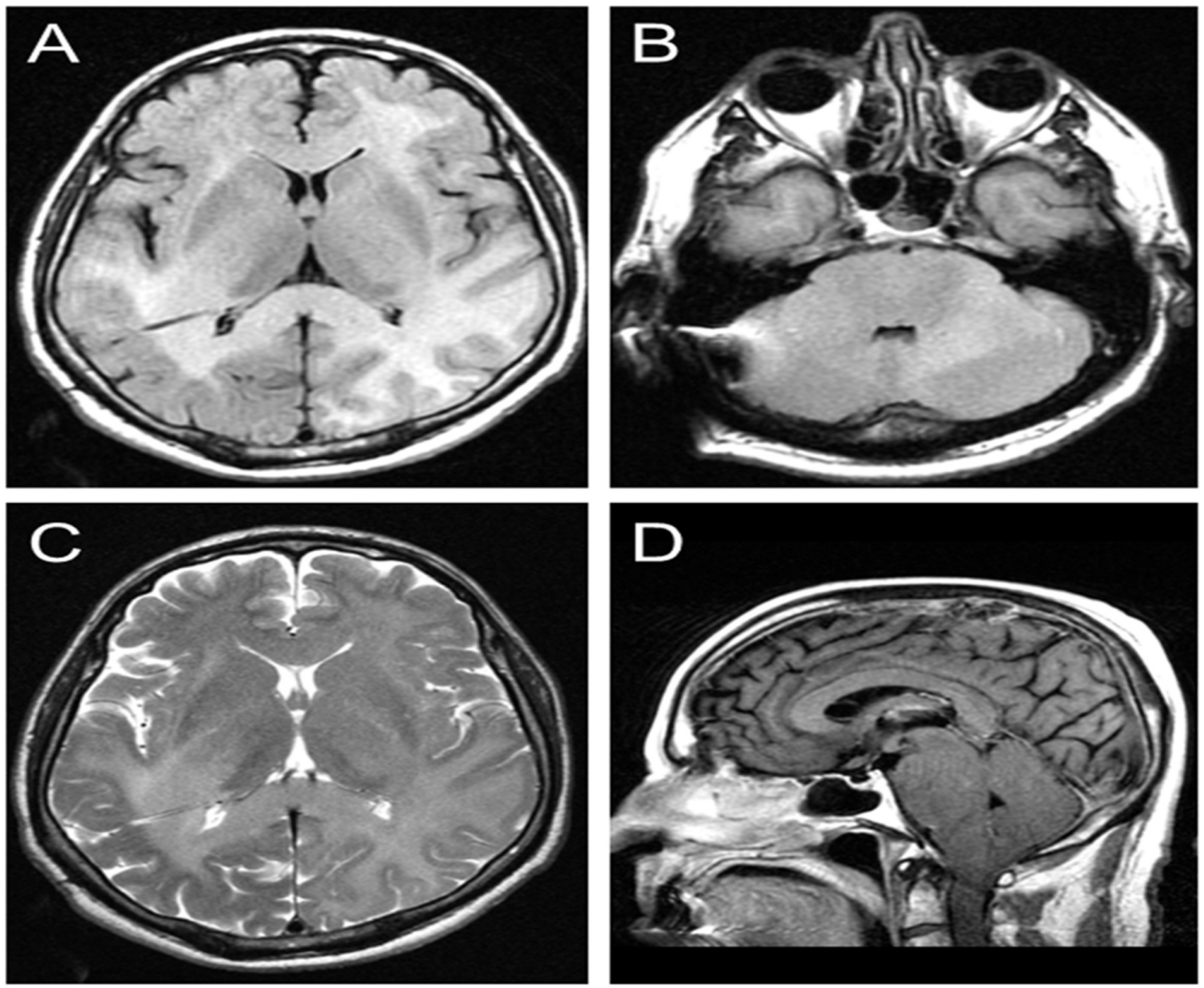

\section{FIGURE 3:}

Follow up images performed approximately 30 months after initial ones performed on presentation and 28 months after right parietal ventriculoperitonal shunt placement. (A) FLAIR MR axial images of supratentorium. Ventricular size remains stable, with no significant change in FLAIR and T2 hyperintensities. (B) FLAIR MR axial images of cerebellum and brainstem now demonstrating increasing FLAIR hyperintensity in left cerebellum. (C) T2 weighted MR images with increasing hyperintesity within bilateral parietotemporal lobes. (D) T1 weighted MR sagittal images demonstrating the increasing fullness of brainstem with increase mass effect within the posterior fossa.

Clinical worsening of the patient's symptoms was consistent with posterior fossa compression. A posterior fossa decompression was performed via a suboccipital craniectomy, C1 laminectomy, and duraplasty. Simultaneously, a second biopsy of a T2 hyperintense area within the left cerebellum was performed. Pathologic findings revealed mild to moderately cellular brain tissue with elongated atypical nuclei. Immunostains demonstrated positivity for S100 and GFAP, with slightly elevated Ki-67 labeling index. Rare nuclei were highlighted by p53. All these findings were consistent with a diffuse infiltrating low-grade astrocytoma. With this pathologic diagnosis, the diagnosis of gliomatosis cerebri was made.

After the posterior fossa decompression, the patient's gait disturbance improved. The patient expired approximately 10 weeks after surgery. He developed respiratory failure, and his family opted for palliative care.

\section{Discussion}

GC is a rare form of brain tumor. Clinical symptoms and imaging findings of GC are variable 
with duration of symptoms. When the disease presents without a focal mass lesion, the diagnosis is often delayed, and the tumor is misdiagnosed as some other neurologic disease. In our case, presenting symptoms suggested a more benign process, such as normal pressure hydrocephalus.

Diverse clinical presentations of GC have been demonstrated in many studies. Jennings, et al. reported that the common neurologic symptoms and signs of GC included corticospinal tract deficits (58\%), dementia (44\%), headache (39\%), seizures (38\%), cranial neuropathies (37\%), papilledema and increased intracranial pressure (ICP) (34\%), and spinocerebellar deficits (33\%) [6]. The major symptom of our 67-year-old patient was mild gait instability.

Although GC has a wide range of presentation age in adults, the majority of patients diagnosed with GC are comparatively young. Taillibert, et al. analyzed 296 individual cases (M/W 169/128) reporting the median age on GC onset is 39 (range: 7 months - 85 years) for men and 45 (range: 1 month - 85 years) for women, citing that GC preferentially occurs in young to middle-aged adults [7]. The age of our patient on initial presentation was 69. Even though the median age of GC presents in a broad range, his GC onset is quite late in comparison to other GC patients.

\section{Pathology}

GC occurs at varying grades and can be either indolent or rapidly progressive [8]. Gutch, et al. reported that the clinical course of GC is typically slow and long, especially when the neoplastic infiltrate is of low histological grade [9].

Histopathologically, GC is characterized by infiltrating neoplastic astrocytic elements. Chen, et al. report that in their cohort, the predominant histopathologic tumor with GC was fibrillary astrocytoma (66.7\%) followed by small cell astrocytoma (5.5\%) [5]. The predominant histopathologic tumor grade in their series was Grade III (67\%), followed by Grade II (21\%), and Grade IV (12\%) [5]. According to Tallibert, et al., astrocytomas were the principal type (108/296 cases) followed by oligodendroglioma (54/296) and oligoastrocytoma (17/296) [7]. One hundred and seventeen of the 296 cases were undetermined/not specified. One hundred of 200 cases were Grade II, followed by Grade III tumors (81/200) [7].

GC can be categorized as primary or secondary where, despite having the same histopathological characteristics, their pattern of development causes their categorization as different entities [6]. Primary GC can be subdivided into two forms: Type 1 and Type 2 GC. Type $1 \mathrm{GC}$ is defined as a diffusely infiltrative glioma without formation of an obvious tumor mass [6]. In contrast, Type 2 GC presents with extensive CNS involvement with diffusely infiltrative glioma, but notably with an associated tumor mass $[6,10]$. Secondary GC is defined as an infiltration of neoplasm that has resulted from the spreading of a focal glioma $[1,6,10]$. In the case of secondary tumors, when it is detected early, local resection of the tumor mass is possible, which may improve the overall prognosis. However, in case of the primary GC, especially Type 1, early detection is difficult as no mass effect is observed on complex imaging studies. MR images of our case demonstrated no obvious tumor mass. Our patient had a left parietal biopsy with pathological diagnosis that was nonspecific. Repeat biopsy performed two and a half years later demonstrated abnormal tissue consistent with diffuse infiltrating low grade astrocytoma, ultimately making the diagnosis of Type 1 primary GC.

\section{Imaging}

MRI studies are considered the gold standard for diagnosing GC. Radiologic features, however, are very nonspecific so that patients are often misdiagnosed with other neurologic diseases. With the current usage of MRI demonstrating diffuse, poorly circumscribed T2-weighted 
hyperintense lesions with associated edema and FLAIR changes of adjacent areas, GC is now more often considered on the differential diagnosis [11]. On T1-weighted MR images, GC is usually seen as isointense to hypointense lesions compared with normal brain [12]. Characteristically, there is no enhancement after the administration of contrast medium. Freund, et al. reported that a large number of different diseases may produce similar changes on CT and MR imaging causing misdirection and, consequentially, misdiagnosis [13]. Such diseases include infectious diseases, leukoencephalopathy, multiple sclerosis, and ischemic disorders. Additionally, the abnormal signals observed on MRI may not fully show the degree of brain invasion [10, 14], which demonstrate that even an abnormal MRI study does not correctly diagnose GC.

\section{Treatment}

Due to the infiltrating character of GC, surgical application is generally limited. Main therapeutic options include radiotherapy, chemotherapy, or a combination of both. Chen, et al. reported a significant association between survival and radiotherapy showing that median progression-free survival was longer by 12 months and median overall survival was longer by 21 months in patients who received radiotherapy [5]. Some studies report that initial chemotherapeutic treatment with temozolomide (TMZ) or PCV (procarbazine, lomustine and vincristine) is effective for some cases of GC [15-17]. TMZ is an oral prodrug that is currently used for the management of malignant gliomas [18]. According to Mattox, et al., patients with GC, who demonstrated a positive response to treatment, were treated by TMZ (temozolomide) (26.2\%), followed by RT alone (26.2\%), concomitant TMZ and RT (20\%), and RT with other agents (PCV, ACNU (Nimustine), vincristine, or cisplatin) (21.3\%) [4]. This study showed that overall survival based on a Kaplan-Meier survival analysis for GC patients with a positive response after chemotherapeutic treatment was 25 months [4]. However, due to limited number of patients who received each therapy and lacking of prospective clinical trials, a guideline for management of GC with chemotherapy and/or radiotherapy has not yet been developed. To predict the benefit of chemotherapy and/or radiotherapy, further clinical prospective trials are needed.

With regard to surgical treatment, a VP shunt was placed in our patient, which relieved symptoms secondary to elevated intracranial pressure. Weston and Lear reported the case of a 44-year-old patient with papilledema [19]. The patient with symptoms related to elevated intracranial pressure underwent shunt implantation; however, it resulted in no improvement in the patient's condition. Later, post-mortem examination revealed that the patient had GC. Rorke-Adams and Portnoy report a pediatric case with GC [20]. Before the diagnosis, a VP shunt was placed in a 13-month-old boy. In this pediatric patient, a posterior fossa decompression (PFD) was performed which relieved symptoms for three months. As the patient's symptoms recurred, a large amount of cerebellar tissue was removed. At the age of 13 years, the patient underwent a shunt revision. At the age of 21 years, when the report was written, the patient is still alive with no radiotherapy or chemotherapy postoperatively. Due to elevated intracranial pressure, a shunt may be placed for symptom relief; however, in the case of GC, the shunt procedure was not a management option. After shunt implant, if the ICP is not under control, GC should be considered as a differential diagnosis.

Our case demonstrates that diagnosis of GC is challenging. To our knowledge, there is no case report describing an elderly patient with primary GC who underwent both a posterior fossa decompression and ventriculoperitoneal shunt placement before initiating chemotherapy and/or radiotherapy. Weinberg, et al. reported three cases of GC in adult patients who subsequently underwent decompression after pursuit of radio- and/or chemotherapies [21]. In their cases, a 50-year-old patient with primary GC and two patients, one a 35-year-old and the other a 40-year-old, with secondary GC underwent posterior fossa decompression once involvement of the tonsillar herniation was detected [21]. In these three cases, PFD has brought 
immediate relief of symptoms. Weinberg, et al. reported that patients with GC can be treated with PFD and duraplasty in the context of palliative management [21].

In our case, due to the marked infiltrative character of the tumor and the negative first biopsy, the diagnosis was not made until two years after the initial presentation. Other differential diagnoses, including paraneoplastic and autoimmune disease, were considered; however, they could not explain the progressive expansion of brainstem and cerebellum. The diagnosis was not made until the second biopsy was performed two years later. It is possible that earlier diagnosis and treatment with radiation therapy and chemotherapy might have prolonged the patient's life and functional independence.

\section{Conclusions}

Patients with GC may present with symptoms of normal pressure hydrocephalus and benefit from ventricular shunting. When brain imaging demonstrates evidence of fullness in the posterior fossa, such patients should be followed carefully for evidence that will allow the diagnosis of GC to be made.

\section{Additional Information \\ Disclosures}

Human subjects: Consent was obtained by all participants in this study. Conflicts of interest: In compliance with the ICMJE uniform disclosure form, all authors declare the following: Payment/services info: All authors have declared that no financial support was received from any organization for the submitted work. Financial relationships: All authors have declared that they have no financial relationships at present or within the previous three years with any organizations that might have an interest in the submitted work. Other relationships: All authors have declared that there are no other relationships or activities that could appear to have influenced the submitted work.

\section{References}

1. Lantos PL, Bruner JM: Gliomatosis cerebri. In Pathology and Genetics of Tumours of the Nervous System. Kleihues P, Cavenee WK (ed): International Agency for Research on Cancer, Lyon, France; 2000. 92-93.

2. Kleihues P, Louis DN, Scheithauer BW, Rorke LB, Reifenberger G, Burger PC, Cavenee WK: The WHO classification of tumors of the nervous system . J Neuropathol Exp Neurol. 2002, 61:215-25.

3. Hecht BK, Turc-Carel C, Chatel M, Lonjon M, Roche JL, Gioanni J, Hecht F, Gaudray P: Chromosomes in gliomatosis cerebri. Genes Chromosomes Cancer. 1995, 14:149-153. 10.1002/gcc.2870140210

4. Mattox AK, Lark AL, Adamson DC: Marked response of gliomatosis cerebri to temozolomide and whole brain radiotherapy. Clin Neurol Neurosurg. 2012, 114:299-306.

10.1016/j.clineuro.2012.01.030

5. Chen S, Tanaka S, Giannini C, Morris J, Yan ES, Buckner J, Lachance DH, Parney IF:

Gliomatosis cerebri: Clinical characteristics, management, and outcomes. J Neurooncol. 2013, 112:267-275. 10.1007/s11060-013-1058-x

6. Jennings MT, Frenchman M, Shehab T, Johnson MD, Creasy J, LaPorte K, Dettbarn WD: Gliomatosis cerebri presenting as intractable epilepsy during early childhood. J Child Neurol. 1995, 10:37-45. 10.1177/088307389501000111

7. Taillibert S, Chodkiewicz C, Laigle-Donadey F, Napolitano M, Cartalat-Carel S, Sanson M: Gliomatosis cerebri: A review of 296 cases from the ANOCEF database and the literature . J Neurooncol. 2006, 76:201-205. 10.1007/s11060-005-5263-0

8. Vates GE, Chang S, Lamborn KR, Prados M, Berger MS: Gliomatosis cerebri: A review of 22 cases. Neurosurg. 2003, 53:261-271. 10.1227/01.NEU.0000073527.20655.E6 
9. Gutch M, Ansari MK, Jain N, Yadav H: A rare case of gliomatosis cerebri presenting as dementia. J Nat Sci Biol Med. 2012, 3:78-80. 10.4103/0976-9668.95973

10. Felsberg GJ, Silver SA, Brown MT, Tien RD: Radiologic-pathologic correlation: Gliomatosis cerebri. AJNR Am J Neuroradiol. 1994, 15:1745-1753.

11. Benjelloun A, Delavelle J, Lazeyras F, Dietrich PY: Possible efficacy of temozolomide in a patient with gliomatosis cerebri. Neurology. 2001, 57:1932-1933. 10.1212/WNL.57.10.1932

12. Shin YM, Chang KH, Han MH, Myung NH, Chi JG, Cha SH, Han MC: Gliomatosis cerebri: Comparison of MR and CT features. AJR Am J Roentgenol. 1993, 161:859-862.

13. Freund M, Hähnel S, Sommer C, Martmann M, Kiessling M, Tronnier V, Sartor K: CT and MRI findings in gliomatosis cerebri: A neuroradiologic and neuropathologic review of diffuse infiltrating brain neoplasms. Eur Radiol. 2001, 11:309-316. 10.1007/s003300000653

14. Koslow SA, Claassen D, Hirsch WL, Jungreis CA: Gliomatosis cerebri: A case report with autopsy correlation. Neuroradiology. 1992, 34:331-333. 10.1007/BF00588195

15. Levin N, Gomori JM, Siegal T: Chemotherapy as initial treatment in gliomatosis cerebri: Results with temozolomide. Neurology. 2004, 63:354-356.

10.1212/01.WNL.0000130249.41341.58

16. Sanson M, Cartalat-Carel S, Taillibert S, Napolitano M, Djafari L, Cougnard J, Gervais H, Laigle F, Carpentier A, Mokhtari K, Taillandier L, Chinot O, Duffau H, Honnorat J, Hoang-Xuan K, Delattre JY, ANOCEF group: Initial chemotherapy in gliomatosis cerebri. Neurology. 2004, 63:270-275. 10.1212/01.WNL.0000129985.39973.E4

17. Herrlinger U, Felsberg J, Küker W, Bornemann A, Plasswilm L,Knobbe CB, Strik H, Wick W, Meyermann R, Dichgans J, Bamberg M, Reifenberger G, Weller M: Gliomatosis cerebri: Molecular pathology and clinical course. Ann Neurol. 2002, 52:390-399. 10.1002/ana.10297

18. Brandes AA, Pasetto LM, Vastola F, Monfardini S: Temozolomide inpatients with high grade gliomas. Oncology. 2000, 59:181-186. 10.1159/000012158

19. Weston P, Lear J: Gliomatosis cerebri or benign intracranial hypertension? . Postgrad Med J. 1995, 71:380-381. 10.1136/pgmj.71.836.380

20. Rorke-Adams LB, Portnoy H: Long-term survival of an infant with gliomatosis cerebelli . J Neurosurg Pediatr. 2008, 2:346-350. 10.3171/PED.2008.2.11.346

21. Weinberg JS, Rhines LD, Cohen ZR, Langford L, Levin VA: Posterior fossa decompression for life-threatening tonsillar herniation in patients with gliomatosis cerebri: report of three cases. Neurosurg. 2003, 52:216-223. 